\title{
VELOCIDADE DE PROPAGAÇÃO DA FRENTE DE DISTÚRBIOS NO MODELO HIDROSTÁTICO DA ATMOSFERA POLITRÓPICA
}

\section{P. Casarin \& V. Kadychnikov}

A atmosfera não contém ondas acústicas na aproximação hidrostática. Entre outras ondas, as ondas de Lamb, que se propagam horizontalmente, são as mais rápidas. A velocidade delas é comparável a do som. O valor exato da velocidade depende da escolha do estado básico da atmosfera usado para a linearização das equações hidrotermodinâmicas. Às condições reais da troposfera da Terra corresponde o estado com a estratificação térmica politrópica. A temperatura deste estado decresce em relação a altura a uma taxa de variação constante $\Gamma$. Supomos que toda a atmosfera tenha uma estratificação politrópica, ou seja, verticalmente finita. Supomos também que a estratificação seja estaticamente estável $\left(\Gamma<\Gamma_{d} \equiv \frac{g}{c_{p}}\right)$ ou neutra $\left(\Gamma=\Gamma_{d}\right)$. O perfil vertical das autofunções é expresso, no modelo politrópico, através das funções de Bessel. Por isso, a equação de dispersão que determina os autovalores do problema e, como conseqüência, o espectro das oscilações da atmosfera, é transcendente. A solução mínima desta equação, isto é, $\Gamma$ o autovalor mínimo corresponde à velocidade máxima de propagação de desvios (velocidade da frente). Quando $\Gamma \approx \Gamma_{d}$ o autovalor mínimo pode ser encontrado facilmente com a ajuda do desenvolvimento da solução em série de potências da pequena diferença $\Gamma_{d}-\Gamma$. É mostrado que uma fórmula analítica simples, obtida mantendo-se na série só os dois primeiros termos, fornece uma boa aproximação não somente para este caso, como também para o caso oposto quando $\Gamma=0$. Uma comparação dos cálculos obtidos pela fórmula analítica e, numericamente, pela equação de dispersão completa mostra uma alta precisão da fórmula em todo o intervalo $0 \leq \Gamma \leq \Gamma_{d}$.

Palavras-chave: Modelo hidrodinâmico; Atmosfera; Estratificação politrópica; Ondas de Lamb.

PROPAGATION SPEED OF THE FRONT OF DISTURBANCES IN A HYDROSTATIC MODEL OF A POLYTROPIC ATMOSPHERE - There are no acoustic waves in a hydrostatic model of the atmosphere. Among the other waves, the most fast ones are the Lamb waves that propagate horizontally. Their speed is comparable with the speed of sound. Its exact value depends on the choice of the basic state of the atmosphere which is used to linearize the hydrothermodynamic equations. It is a state with a polytropic stratification where the temperature decreases linearly with altitude at a constant lapse-rate $\Gamma$ that corresponds to the actual conditions of the Earth's troposphere. It is assumed that all the atmosphere has a politropic stratification, i.e., it is finite with altitude because $\Gamma>0$. We suppose also that the stratification is statically stable $\left(\Gamma<\Gamma_{d} \equiv \frac{g}{c_{p}}\right)$ or neutral $\left(\Gamma=\Gamma_{d}\right)$. The vertical profile of the eigenmodes in the polytropic model is described with the help of the Bessel functions. Therefore, the dispersion equation that determines the eigenvalues of the problem, and consequently an oscillation spectrum, is transcendental. The highest propagation speed (the front speed) corresponds to the minimum solution of this equation, i.e., the minimum eigenvalue. When $\Gamma \approx \Gamma_{d}$, the minimum eigenvalue can be calculated using the development of the solution into the series by powers of the small difference $\Gamma_{d}-\Gamma$. It is proved that a simple analytical formula, obtained by keeping in the 
series only the two first terms, gives a good approximation not only of this limit but of the opposite limit when $\Gamma=0$, too. A comparison of the results obtained by the analytical formula and using the complete dispersion equation shows a good precision of the formula in the whole range of $0 \leq \Gamma \leq \Gamma_{d}$.

Key words: Hydrodynamic model; Atmosphere; Polytropic stratification; Lamb waves.

\author{
Universidade Federal de Pelotas \\ Centro de Pesquisas Meteorológicas \\ Avenida Ildefonso Simões Lopes, 2751 \\ 96.060-290 - Pelotas - RS \\ Fax: (0532) 23-48-14 \\ Telefone: (0532) 23-47-88 \\ E-mail: casarin@urano.cpmet.ufpel.tche.br
}

\section{INTRODUÇÃO}

$\mathrm{Na}$ construção de modelos atmosféricos numericamente estáveis, um parâmetro importante que garante uma escolha ótima do passo de tempo, em dependência do tamanho dos passos espaciais da grade de um esquema numérico, é o de Courant (Marchuk, 1974; Mesinger \& Arakawa, 1976). O parâmetro relaciona estes passos com a velocidade de propagação do sinal no meio, i.e., com a velocidade da frente dos desvios. Em modelagens de processos atmosféricos de escala grande são usados alguns modelos hidrostáticos (Holton, 1992; Marchuk, 1974). Os modelos filtram as ondas acústicas rápidas que se propagam praticamente na direção vertical (Holton, 1992). Elas não estão relacionadas com os processos atmosféricos de grande escala. Nos modelos hidrostáticos nenhuma frente se propaga verticalmente e um passo de tempo admissível, do ponto de vista da estabilidade do esquema numérico, pode depender só do passo horizontal da grade e da velocidade horizontal dos desvios. Esta velocidade é uma soma da velocidade do vento e da de grupo das ondas mais rápidas. Tais ondas, no modelo hidrostático, são as de Lamb (Gill, 1982). A velocidade horizontal destas ondas é comparável a velocidade do som. A velocidade de propagação da energia pelas ondas internas gravitacionais e de Rossby é muito menor.

A velocidade de propagação das ondas de Lamb na atmosfera depende da escolha do estado básico da atmosfera em relação ao qual se faz a linearização das equações hidrotermodinâmicas. Três parâmetros termodinâmicos do estado básico (pressão, densidade e temperatura) estão relacionados pelas equações da hidrostática e do estado. A terceira equação pode ser escolhida de muitas maneiras. A escolha mais simples, mais frequentemente usada, é a de uma atmosfera isotérmica (Gill, 1982; Houghton, 1977). Nesse caso, as amplitudes das autofunções da atmosfera obedecem a equações ordinárias com alguns coeficientes constantes. Por isso, o perfil vertical delas tem uma forma exponencial-ondulatória e a equação de dispersão, que determina o espectro das oscilações, isto é, a dependência da freqüência em relação ao comprimento de onda, tem uma forma algébrica.

Em outra escolha de um estado básico, os perfís verticais das autofunções, mesmo na atmosfera homogênea, sem simplificações adicionais, obedecem a equações mais complexas (Holton, 1992).

No modelo não hidrostático, que é usado para a descrição dos processos de propagação das ondas acústico-gravitacionais na atmosfera, isto pode ser, no caso do estado politrópico, uma equação confluente hipergeométrica (Pekeris, 1948). No caso de um estado com um perfil vertical mais complicado, isto pode ser uma equação ainda mais complexa tal como uma hipergeométrica comum (Nalesso \& Jacobson, 1995).

Um modelo politrópico da atmosfera, isto é, com decréscimo linear da temperatura com a altura, corresponde bem às condições reais da parte inferior da atmosfera, a troposfera que se estende do solo até a tropopausa. No modelo hidrostático, que é usado para uma descrição do tempo, o perfil das autofunções se expressa através das funções de Bessel. A equação de dispersão é transcendente. É interessante, sob o 
ponto de vista teórico e também prático, procurar uma fórmula simples que descreva a velocidade de propagação dos desvios com dependência da taxa de variação vertical da temperatura em toda a região $0<\Gamma<\Gamma_{d}$, tendo em vista que os valores da velocidade nos pontos extremos $\Gamma=0$ (atmosfera isotérmica) e $\Gamma=\Gamma_{d}$ (atmosfera com estratificação neutra) são conhecidos (Holton, 1992).

\section{EQUAÇÃO BÁSICA}

As equações hidrotermodinâmicas da atmosfera hidrostática podem ser escritas no sistema das coordenadas isobáricas como:

$$
\begin{aligned}
& \frac{d u}{d t}+\phi_{x}=f v, \quad \frac{d v}{d t}+\phi_{y}=-f u, \quad \varsigma \phi_{\varsigma}=-R T, \\
& u_{x}+v_{y}+\omega_{\varsigma}=0, \quad \frac{d T}{d t}=\frac{R T \Gamma_{d}}{g \varsigma} \omega,
\end{aligned}
$$

onde $t$ é o tempo; $x, y, \varsigma=\frac{P}{P} \quad$ são as coordenadas espaciais ( $p$ - pressão, $P=1000 h P a$ ); $u, v, \omega$ são as velocidades correspondentes; $\phi$ é o geopotencial; $T$ é a temperatura; $f$ é o parâmetro de Coriolis; $R$ é a constante dos gases para o ar; $g$ é a aceleração da gravidade; $\Gamma_{d}=\frac{g}{c_{p}}$ é o gradiente adiabático seco e $C_{p}$ é o calor específico a pressão constante (Holton, 1992).

Linearizando as equações em relação ao estado politrópico em repouso, a saber:

$$
\bar{u}=\bar{v}=\bar{\omega}=0, \quad \bar{T}=\bar{T}_{1}-\Gamma z,
$$

onde $\bar{T}_{1}$ é o valor médio da temperatura junto do solo, em $\varsigma=1$, e $\Gamma$ é o gradiente vertical da temperatura $\bar{T}$, obtemos as equações para os desvios $u^{\prime}, v^{\prime}, \omega^{\prime}, \phi^{\prime}, T^{\prime}$. Omitindo as linhas temos

$$
\begin{aligned}
& u_{t}+\phi_{x}=f v, \quad v_{t}+\phi_{y}=-f u, \quad \varsigma \phi_{\varsigma}=-R T, \\
& u_{x}+v_{y}+\omega_{\varsigma}=0, \quad T_{t}=\frac{R \bar{T}_{1}\left(\Gamma_{d}-\Gamma\right)}{g \varsigma^{1-\alpha}} \omega,
\end{aligned}
$$

onde $\alpha=\frac{R \Gamma}{g} \quad$ e o parâmetro $f$ considera-se constante.

Vamos supor que em coordenadas horizontais o problema seja periódico. Em relação à altura o sistema tem duas derivadas. Por isso, é preciso colocar duas condições de contorno. Como primeira condição, exigimos que a velocidade vertical $w \equiv \frac{d z}{d t}$ seja zero no solo. Assim $\left.\frac{d \phi}{d t}\right|_{\varsigma=1}=0$ ou no caso linear $\phi_{t}+\bar{\phi}_{\varsigma} \omega=0$. Usando a equação hidrostática em (2) temos:

$$
\phi_{t}=R \bar{T}_{1} \omega, \quad \text { quando } \varsigma=1 .
$$

A segunda condição é escolhida das razões energéticas. De (2) obtem-se uma lei de conservação da energia na seguinte forma:

$$
\begin{gathered}
\frac{\partial}{\partial t} \frac{1}{2}\left[u^{2}+v^{2}+\frac{g}{\overline{T_{1}}\left(\Gamma_{d}-\Gamma\right) \varsigma^{\alpha}} T^{2}\right]+ \\
+(u \phi)_{x}+(v \phi)_{y}+(\omega \phi)_{\varsigma}=0
\end{gathered}
$$

Integrando esta equação numa região periódica em $x, y$ e de 0 até 1 em $\varsigma$ vemos que, de acordo com a condição de contorno (3) para $\varsigma=1$, a integral espacial de uma forma quadrática, a saber,

$$
E=\frac{1}{2}\left[u^{2}+v^{2}+\frac{g}{\bar{T}_{1}\left(\Gamma_{d}-\Gamma\right) \xi^{\alpha}} T^{2}+\frac{1}{R \bar{T}_{1}} \phi_{1}^{2}\right],
$$

conserva-se no tempo se

$$
\omega \phi=0, \quad \text { quando } \varsigma=0 .
$$

Esta será nossa segunda condição de contorno.

Vamos procurar soluções do sistema (2) sob as condições (3) e (4) na forma das ondas:

$$
\begin{aligned}
& \psi(x, y, \varsigma, t)=\hat{\psi}(\varsigma) \exp i(k x+l y-\lambda t), \\
& (\psi \equiv u, v, \omega, \phi, T),
\end{aligned}
$$

com amplitudes que dependem de $\varsigma$ pois os coeficientes das equações dependem desta coordenada. Colocando (5) nas Eqs. (2) e excluindo todas as amplitudes, exceto $\hat{\phi}$, obtemos a seguinte equação de segunda ordem:

$$
\frac{d}{d \varsigma}\left(\varsigma^{2-\alpha} \frac{d \hat{\phi}}{d \varsigma}\right)+\frac{R^{2} \overline{T_{1}}\left(\Gamma_{d}-\Gamma\right)}{g} \frac{\rho^{2}}{\lambda^{2}-f^{2}} \hat{\phi}=0,
$$

onde $\rho^{2}=k^{2}+l^{2}$. Como as amplitudes da velocidade vertical e do geopotencial estão relacionadas pela fórmula 


$$
\hat{\omega}=i \frac{\lambda g}{R^{2} \bar{T}_{1}\left(\Gamma_{d}-\Gamma\right)} \varsigma^{2-\alpha} \hat{\phi}_{\varsigma}
$$

as condições de contorno (3) e (4) ficam respectivamente:

$$
\begin{aligned}
\frac{g}{R\left(\Gamma_{d}-\Gamma\right)} \hat{\phi}_{\varsigma}+\hat{\phi}=0, & \text { quando } \varsigma=1 \\
\varsigma^{2-\alpha} \hat{\phi} \hat{\phi}_{\varsigma}=0, & \text { quando } \varsigma=0
\end{aligned}
$$

\section{EQUAÇÃO DE DISPERSÃO}

A Eq. (6) é do tipo de Bessel e a sua solução geral é (Watson, 1966):

$$
\hat{\phi}(\varsigma)=\varsigma^{\frac{-1+\alpha}{2}}\left[C_{1} J_{\frac{1-\alpha}{\alpha}}\left(Z \zeta^{\frac{\alpha}{2}}\right)+C_{2} N_{\frac{1-\alpha}{\alpha}}\left(Z \varsigma^{\frac{\alpha}{2}}\right)\right],
$$

onde $J$ e $N$ são as funções de Bessel de primeiro e segundo tipo respectivamente, $C_{1}$ e $C_{2}$ são constantes arbitrárias e o parâmetro

$$
Z=\frac{2 R \rho}{\alpha} \sqrt{\frac{\overline{T_{1}}\left(\Gamma_{d}-\Gamma\right)}{g\left(\lambda^{2}-f^{2}\right)}} .
$$

Quando $x \rightarrow 0$ as funções $J_{v}(x) \sim x^{v}$, $N_{v}(x) \sim x^{-v}$, então somente a primeira solução em (9) cumpre a condição superior (8), isto é, $C_{2}=0$. A solução rejeitada não cumpre também a condição de limitação da integral espacial da forma quadrática $E$. Assim uma solução da Eq. (6) sob a condição (8), a menos de um fator arbitrário, é

$$
\hat{\phi}(\varsigma)=\varsigma^{\frac{-1+\alpha}{2}} J_{\frac{1-\alpha}{\alpha}}\left(Z \varsigma^{\frac{\alpha}{2}}\right) .
$$

Colocando-a na condição de contorno inferior (7) obtemos a equação de dispersão na forma:

$$
\frac{a}{1-a} \frac{Z}{2} J_{b+1}(Z)=J_{b}(Z)
$$

onde as denotações:

$$
a=\frac{\Gamma}{\Gamma_{d}}, \quad b=\frac{1-\alpha}{\alpha}
$$

são usadas.

Depois dos autovalores do problema, $\mathrm{Z}_{n}$, da equação de dispersão (12), serem encontrados, a freqüência $\lambda$ será determinada pela fórmula (10), de onde segue que

$$
\lambda_{n}^{2}=R \overline{T_{1}} \rho^{2} \frac{\gamma}{\gamma-1} \cdot \frac{1-a}{a^{2}} \frac{1}{\left(\frac{Z_{n}}{2}\right)^{2}}+f^{2},
$$

onde $\gamma=\frac{c_{p}}{c_{v}}, R=c_{p}-c_{v}$.

A velocidade de propagação da frente dos desvios, $C$, é igual a max $\frac{\partial \lambda}{\partial \rho}$. O valor máximo, de acordo com (14), é atingido quando $\rho \rightarrow \infty$, pois $\frac{\partial}{\partial \rho}\left(\frac{\partial \lambda}{\partial \rho}\right)>0$.

Assim

$$
C=\sqrt{R \overline{T_{1}} \frac{\gamma}{\gamma-1} \cdot \frac{1-a}{a^{2}} / \delta}
$$

onde

$$
\delta=\min _{n}\left(\frac{Z_{n}}{2}\right)^{2} .
$$

Então, a nossa tarefa é encontrar uma solução mínima da Eq. (12) porque, de acordo com (13), o parâmetro $b$ também depende do parâmetro $a$ :

$$
b=\frac{\gamma}{\gamma-1} \frac{1}{a}-1
$$

\section{DEDUÇÃO DE UMA FÓRMULA PARA A VELOCIDADE DE PROPAGAÇÃO DA FREN- TE}

Usando as séries para a apresentação das funções $J_{b}(\mathrm{Z})$ e $J_{b+1}(\mathrm{Z})$, a Eq. (12) pode ser escrita na forma (Watson, 1966):

$$
\begin{aligned}
& \frac{a}{1-a} \frac{\delta}{b+1}\left[1-\frac{\delta}{1 !(b+2)}+\frac{\delta^{2}}{2 !(b+2)(b+3)}-\frac{\delta^{3}}{3 !(b+2)(b+3)(b+4)}+\ldots\right]= \\
& =1-\frac{\delta}{1 !(b+1)}+\frac{\delta^{2}}{2 !(b+1)(b+2)}-\frac{\delta^{3}}{3 !(b+1)(b+2)(b+3)}+\ldots
\end{aligned}
$$

Quando $a \rightarrow 1$ o valor de $b$ fica limitado, como mostra a definição (16). Por isso, de acordo com (17), o valor de $\delta$ tende a zero. Denotamos como $\delta^{(1)}$, a solução que corresponde a este limite, quando em (17) são mantidos um termo a esquerda e a direita. Temos:

$$
\delta^{(1)}=\frac{(b+1)(1-a)}{a}=\frac{\gamma}{\gamma-1} \cdot \frac{1-a}{a^{2}} .
$$

A solução pode tornar-se mais precisa com a ajuda 


\begin{tabular}{c|cccc}
$A$ & $\delta^{(1)}$ & $\delta^{(2)}$ & $\delta^{(3)}$ & $\delta$ \\
\hline 0,1 & 315,00 & 236,25 & 237,31 & 238,10 \\
0,5 & 7,0000 & 6,1250 & 6,1493 & 6,1528 \\
0,9 & 0,43210 & 0,42228 & 0,42235 & 0,42236
\end{tabular}

Tabela 1- Dependência das diferentes aproximações de $\delta$ em relação aos parâmetro $\alpha$.

Table 1 - Dependence of distinct approximation of $\delta$ in relation to $\alpha$.

do seu desenvolvimento em série de potências do parâmetro pequeno $\delta^{(1)}$. Vamos escrever os três primeiros termos:

$$
\delta=\delta^{(1)}-\frac{\left(\delta^{(1)}\right)^{2}}{(b+1)(b+2)}+\frac{2\left(\delta^{(1)}\right)^{3}}{(b+1)^{2}(b+2)^{2}(b+3)}+\ldots
$$

Acontece que a soma finita (19), sem termos em vista os outros termos da série, pode ser usada para obter uma boa aproximação para o parâmetro $\delta$ também no caso do limite oposto $a \rightarrow 0$. Na realidade, quando $a \rightarrow 0$, de acordo com as definições (16) e (18), $\delta^{(1)} \sim a^{-2}$ e os valores $b+1, b+2, \ldots \sim \mathrm{a}^{-1}$, isto é, o terceiro termo em (19) é pequeno em comparação aos dois primeiros termos cujas ordens são as mesmas. Todos os outros termos omitidos em (19) são ainda menores.

A Tab. 1 mostra o caráter da dependência do parâmetro $0<a<1$ dos valores: $\delta^{(1)}, \delta^{(2)}$ (as duas primeiras parcelas em (19)), $\delta^{(3)}$ (as três parcelas em (19)) e $\delta$ (a solução numérica da equação (17)).

Para $a \rightarrow 1$ todos os quatro valores $\delta^{(1)}, \delta^{(2)}$, $\delta^{(3)}$ e $\delta$ são iguais a expressão (18) e por isso o radicando em (15) torna-se $R \bar{T}_{1}$. Para $a \rightarrow 0$ o valor $\delta^{(1)}$ é (18) e o radicando em (15) torna-se novamente $R \bar{T}_{1}$. Mas os valores $\delta^{(2)}, \delta^{(3)}$ e $\delta$ nesse caso, como pode ser visto, são $\frac{\delta^{(1)}}{\gamma}$. Por isso, para estes três valores, o radicando em (15) fica $\gamma R \bar{T}_{1}$.

Da Tab. 1 vemos que o valor

$$
\delta^{(2)}=\delta^{(1)}\left[1-\frac{\delta^{(1)}}{(b+1)(b+2)}\right]
$$

já praticamente coincide com a solução exata em toda a região $0 \leq a \leq 1$.

Usando a aproximação $\delta=\delta^{(1)}$ temos de (15), para a velocidade da frente, o valor $\sqrt{R \bar{T}_{1}}$ que não depende de $a$. A aproximação $\delta=\delta^{(2)}$ transforma a fórmula (15) em

$$
C=\sqrt{\frac{a(\gamma-1)+\gamma}{2 a(\gamma-1)+1} R \bar{T}_{1}} .
$$

O multiplicador na frente de $R \bar{T}_{1}$ é uma função monótona de $a$. Como já foi dito, para uma atmosfera com estratificação neutra $(a=1)$, ele é igual a 1 e para uma atmosfera isotérmica $(a=0)$ é $\gamma$. Ambos valores limitantes são bem conhecidos (Holton, 1992). A fórmula (20) fornece uma possibilidade de calcular a velocidade máxima de propagação dos desvios na atmosfera politrópica para todos os valores do parâmetro da estratificação $a=\Gamma / \Gamma_{d}$ dentro do intervalo $0 \leq a \leq 1$.

\section{CONCLUSÃO}

No modelo politrópico da atmosfera hidrostática, isto é, com o perfil (1) da temperatura, o perfil vertical das autofunções (11) se expressa através das funções de Bessel. Por isso a equação de dispersão (12) (ou na forma (17)) que determina os autovalores do problema e, por consequiência, o espectro das oscilações $\lambda(\rho)$, é transcendente. No caso da estratificação da atmosfera ser próxima à neutra $\left(a \equiv \frac{\Gamma}{\Gamma_{d}} \approx 1\right)$, o autovalor mínimo do problema que corresponde à velocidade máxima de propagação da energia dos desvios (velocidade da frente) pode ser encontrado facilmente desenvolvendo a solução em série (19) pelo parâmetro pequeno 1- $a$. O primeiro termo da série fornece, para a velocidade da frente, um valor independente de $a$. Ele corresponde ao valor exato da velocidade na atmosfera com a estratificação neutra. A adição de mais um termo leva-nos para uma fórmula analítica simples (20) para descrever a dependência da velocidade em relação ao parâmetro $a$. Está provado que é preciso ter em vista os mesmos dois termos da série (e por isso está válida a mesma fórmula analítica) também no caso do limite oposto $a \rightarrow 0$. Uma comparação dos resultados obtidos pela fórmula (20), que descreve bem os dois casos limitantes, com os resultados da solução numérica da equação de dispersão completa (12) mostra que a diferença entre 
eles em toda a região $0 \leq a \leq 1$ não excede $1 \%$. Então a fórmula (20) é válida para todas as estratificações estáveis da atmosfera politrópica.

\section{REFERÊNCIAS}

GILL, A.E. - 1982 - Atmosphere - Ocean Dynamics, London, Academic Press, Inc., 662p.

HOLTON, J.R. - 1992 - An Introduction to Dynamic Meteorology, New York, Academic Press, 509p.

HOUGHTON, J.T. - 1977 - The Physics of Atmospheres, Cambridge University Press, 203p.

MARCHUK, G.J. - 1974 - Numerical Methods in Weather Prediction, London, Academic Press,
$277 \mathrm{p}$.

MESINGER F. \& ARAKAWA A. - 1976 Numerical Methods Used in Atmospheric Models, GARP Publ. series, $N^{\circ} 17,65 \mathrm{p}$.

NALESSO, G.F. \& JACOBSON A.R. - 1995 Acoustic-gravity waves impulse response to a point source in a nonisothermal stratified atmosphere, $\mathrm{J}$. of Geoph. Res. 100 (A.6), 9741-9751.

PEKERIS, C.L. - 1948 - The propagation of a pulse in the atmosphere, II, Phys. Review, ser. 2, 73, $145-154$.

WATSON, G.N. - 1966 - A treatise on the Theory of Bessel Functions, Cambridge University Press, 804p.

\section{PROPAGATION SPEED OF THE FRONT OF DISTURBANCES IN A HYDROSTATIC MODEL OF A POLYTROPIC ATMOSPHERE}

Hydrostatic models of the atmosphere are commonly used in the hydrodynamic modelling of large-scale atmospheric processes. The models do not include acoustic waves that propagate vertically with the sound speed and which are not related to the weather processes. Therefore, a time step in finite difference models can not depend on the vertical model resolution. Its dependence on the horizontal resolution of explicit numerical schemes is determined by the Courant numerical stability condition. This condition relates the space and time steps of the schemes with the speed of the signal propagation in the environment. The propagation speed is equal to the maximum of the group velocity of all wave types (Lamb, gravity and Rossby waves), in a hydrostatic model of the atmosphere, and in all wavelengths. The most fast waves in the hydrostatic model are the Lamb waves. The speed of their energy propagation in all wavelengths is comparable with the sound speed. However, the exact value of the speed depends on the choice of the basic state of the atmosphere. Usually, waves in an isothermal atmosphere are examined and their propagation speeds are evaluated. The advantage of this model is that it is the unique model where the vertical profile of eigenmodes, without any further simplification, is described by the equations with constant coefficients and, hence, the profile is wavy-exponential. However, a state with a linear distribution of the temperature with altitude corresponds better to the actual conditions in the troposphere, where the temperature decreases from the ground up to the tropopause approximately with the constant lapse rate $\Gamma$. We suppose all the atmosphere to be stratified in the same form. In this case, the vertical profile of the eigenmodes is described with the help of the Bessel functions. The condition that the spatially integrated energy of the perturbations is conserved in time, leads up to a transcendental dispersion equation for the eigenvalues of the problem. They determine the oscillation spectrum. The speed of the propagation signal in the model is defined by the minimum eigenvalue. In order to find this eigenvalue, it is used in the equation the development of the solution into the series by powers of the difference $\Gamma_{d}-\Gamma$. If the difference is small, the minimum solution of the dispersion equation can be represented with the help of the two first terms of the series by a simple analytical formula. Hence, the propagation speed (the front speed) is expressed through the parameter $\Gamma$ by an analytical formula, too. It is shown that the same formula is valid in the opposite limiting case when $\Gamma=0$. The solutions for both $\Gamma=\Gamma_{d}$ and $\Gamma=0$ coincide with the corresponding exact solutions which are well known from the literature. A comparison of the results obtained by the analytical formula and, numerically, from the complete dispersion equation shows that errors on the formula do not exceed $1 \%$ in the whole range of $\Gamma$ variations between 0 and $\Gamma_{d}$.

(See note about the authors in page 207) 